\title{
Estimación de la genotoxicidad del río Sinú mediante un bioensayo con Allium cepa I. en Montería, Córdoba-Colombia
}

\section{Estimation of the genotoxicity of the Sinú river through to bioassay with Allium cepa I. in Montería, Córdoba-Colombia}

\section{Teodora Cavadía M.; María Roche M.; Rosalyn Romero A}

Universidad de Córdoba, Facultad de Ciencias Básicas, Programa de Biología, Montería- Colombia.

\section{Resumen}

Las alteraciones son producidas por desechos tóxicos en ambientes contaminados, siendo el ecosistema acuático afectado por vertimientos de estos en su lecho. La implementación de Allium cepa L. como organismo de ensayo es útil para estudios genotóxicos. Esta investigación tuvo como finalidad, estimar la genotoxicidad en muestras de agua del rio Sinú de la ciudad de Montería. Las muestras fueron colectadas en la Vereda Jaraquiel, Avenida Primera y Los Garzones. Luego se realizó el ensayo Allium test, el cual consistió en seleccionar 16 bulbos de cebolla que fueron introducidos en las muestras problema y control. Después de $72 \mathrm{~h}$ se cortaron las raíces tiernas y se sumergieron en Aceto-Orceina por $48 \mathrm{~h}$. Posteriormente se llevaron al microscopio, efectuándose un conteo celular y se observaron las anomalías cromosómicas. Se hicieron pruebas de normalidad y análisis de varianza ANOVA. Los índices mitóticos de las fases mostraron diferencias significativas $(\mathrm{P}=0.0440)$ y presencia de alteraciones en las muestras problema, presentando Los Garzones el mayor número de alteraciones en la división celular en la etapa anafase con puente, presencia de micronúcleos y otras. Se evidenció genotoxicidad en los puntos estudiados por la influencia de contaminación en el rio Sinú.

Palabras clave: Allium test, alteraciones, células, contaminación, río Sinú

\section{Abstract}

The alterations are produced by toxic waste in contaminated environments, being the aquatic ecosystem affected by dumping in river. The implementation of Allium cepa L. as a test organism is useful for genotoxic studies. The purpose of this research was to 
estimate genotoxicity in water samples from the Sinú river in the city of Montería. The samples were collected in the Vereda Jaraquiel, Avenida Primera and Los Garzones. Then the Allium test was carried out, which consisted of selecting 16 onion bulbs that were introduced into the problem and control samples. After $72 \mathrm{~h}$ the tender roots were cut and submerged in Aceto-Orceina for $48 \mathrm{~h}$. Subsequently they were taken to the microscope, carrying out a cell count and chromosomal abnormalities were observed. Normality tests and ANOVA analysis of variance were made. The mitotic indices of the phases showed significant differences $(P=0.0440)$ and presence of alterations in the problem samples, Los Garzones presenting the largest number of alterations in cell division in the anaphase stage with bridge, presence of micronuclei and others. Genotoxicity was evidenced in the points studied due to the influence of pollution in the Sinú river.

Key words: Allium test, alterations, cells, contamination, Sinú river.

\section{Introducción}

Las alteraciones que se producen por sustancias tóxicas en ambientes contaminados afectan el material genético de los organismos expuestos, evidentemente el ecosistema acuático por su fácil acceso de vertimiento de desechos es afectado, siendo estos de tipo industrial, doméstico, agrícola, entre otros, influenciado por el desarrollo de ciudades aledañas a los ríos ${ }^{1}$.

La ciudad de Montería se caracteriza por poseer terrenos fértiles que son utilizados para la producción agrícola y ganadera favorecida por el río Sinú2, donde el uso de fertilizantes y plaguicidas llegan al río. El río Sinú atraviesa la ciudad de extremo a extremo, siendo esta fuente hídrica propicia para vertimientos de sustancias contaminantes ${ }^{3}$, causando efectos en este ecosistema, sin embargo, la falta de estudios que muestren las consecuencias que tienen estos a nivel genético son escasos.

La implementación de pruebas biológicas usadas en la seguridad de la calidad ambiental, entre las cuales se encuentran los ensayos de genotoxicidad y mutagenicidad en una amplia gama de organismos, han sido de gran ayuda para la realización de muchos trabajos ${ }^{4}$.

Una de las pruebas más utilizadas es el ensayo Allium test, siendo Allium cepa un organismo importante para estudios genotóxicos, por su sensibilidad a sustancias tóxicas o contaminantes cuando su material genético está expuesto a dichas sustancias, sufriendo alteraciones cromosómicas, cambios en los procesos de división celular, mutagénesis 
entre otras, prueba empleada en el monitoreo ambiental ${ }^{5}$. Por lo tanto, este trabajo tuvo como objetivo estimar la genotoxicidad en muestras de agua del río Sinú en la ciudad de Montería, utilizando el bioensayo Allium test.

\section{Materiales y métodos}

Área de estudio. El área de estudio correspondió al río Sinú en la ciudad de Montería, Córdoba-Colombia (Figura 1). Los puntos de muestreo se ubicaron en la Vereda Jaraquiel ( $\left.8^{\circ} 41^{\prime} 41.7^{\prime \prime} \mathrm{N} 75^{\circ} 56^{\prime} 56.3^{\prime \prime} \mathrm{W}\right)$, la Avenida Primera $\left(8^{\circ} 45^{\prime} 49.0^{\prime \prime} \mathrm{N} 75^{\circ} 53^{\prime} 04.7^{\prime \prime} \mathrm{W}\right)$ y Los Garzones- Boca de la Ceiba ( $\left.8^{\circ} 49^{\prime} 41.0^{\prime \prime} \mathrm{N} 75^{\circ} 51^{\prime} 22.5^{\prime \prime} \mathrm{W}\right)$.

Recolección de las muestras. Las muestras de agua de cada zona fueron colectadas en frascos de $1000 \mathrm{ml}$ previamente esterilizados en Autoclave del Laboratorio de Genética de la Universidad de Córdoba y fueron llevadas al mismo para su estudio. Las muestras de agua se depositaron en frascos de $125 \mathrm{ml}$ previamente esterilizados.

Técnica Allium test. Se seleccionaron 16 bulbos de cebolla de apariencia, tamaño y forma similar, con un bisturí se les retiro la parte radicular, luego se introdujeron 4 bulbos en frascos de $125 \mathrm{ml}$ para cada muestra de agua, además se utilizó agua destilada como muestra control, dejándose 4 días en un lugar fresco del laboratorio de genética, transcurrido este tiempo se retiró el ápice de las raíces tiernas seleccionando 2 de cada bulbo, siendo sumergidos los ápices en aceto-orceina durante 48 horas en tubos de ensayo ${ }^{6}$. Los ápices de cebolla se colocaron en portaobjetos con cubreobjetos produciendo un squash y fueron observadas al microscopio óptico marca Leica, modelo DM500 en objetivo de 100x.

Índice Mitótico (IM). Se realizó un conteo total de mil células en cada muestra y con base a ello se calculó el índice mitótico como el número de células en división sobre el número total de células observadas, expresadas en porcentaje aplicando la siguiente formula:

$$
I M=\frac{\# \text { de celulas en divison }}{\# \text { de celulas totales }} \times 100
$$

Así mismo se analizó el índice de fases a partir del número de células de cada fase sobre el número total de células observadas en división, expresados en porcentajes aplicando la siguiente formula:

$$
I F=\frac{\# \text { de celulas de cada fase }}{\# \text { de celulas en divison }} \times 100
$$


Identificación de anomalías. Se observó al microscopio la presencia de alteraciones en división celular y se realizó un conteo total para cada muestra problema de las alteraciones presentes, se tomaron registros fotográficos en microscopio óptico Carl Zeiss, Axiostar Plus, Alemania, realizados en el Laboratorio de Microscopía del Centro de Investigación Piscícola CIMPIC de la Universidad de Córdoba.

Análisis estadístico. Se realizaron pruebas de normalidad, a través de la prueba de distribución normal Shapiro-Wilk y homogeneidad de varianzas, con el fin de establecer la ruta de análisis más apropiada; ANOVA para datos paramétricos y Kruskal-Wallis para datos no paramétricos.

Al encontrarse diferencias estadísticamente significativas entre los conjuntos de datos de los índices mitóticos y las alteraciones, se aplicó la prueba de múltiples rangos (LSD), utilizando el software estadístico Stagraphics Centurión XVI.I. Los datos se organizaron en tablas realizadas con el programa Microsoft Excel 2010.

\section{Resultados}

\section{Índice mitótico}

Los valores de los índices mitóticos se observan en la Tabla 1. mostrando que la muestra control reportó los mayores valores (9.36\%) en comparación con las muestras problema Vereda Jaraquiel y Los Garzones (6.66\% y $7.73 \%)$, el índice de fases en la muestra control mostró valores de $16.95 \%$ en metafase y $19.82 \%$ en anafase, valores menores en comparación con las muestras problema Avenida primera con $27.66 \%$ en metafase, y $21.3 \%$ en anafase y en Los Garzones con un $26.28 \%$ en metafase, y $24.03 \%$ en anafase. El índice metafase para la prueba ANOVA $\left(F_{(11)}: 4,30 ; P: 0,0440\right)$, reveló diferencias significativas en las muestras, y al aplicar la prueba de múltiples rangos (LSD) se evidenció diferencias entre la muestra control y las muestras de la Avenida primera y Los Garzones (Tabla 1).

\section{Alteraciones celulares}

Se observaron variaciones en la Metafase con alteración numérica y Anafase irregular, con rotura cromosómica en todas las muestras estudiadas, presentándose en Los Garzones la mayor cantidad de alteraciones mitóticas, micronúcleos y células con doble núcleo, ausentes en las zonas Vereda Jaraquiel y Avenida Primera como se indica los valores en la Tabla 2.

\section{Discusión}


Los índices mitóticos de las muestras problemas Vereda Jaraquiel y Los Garzones resultaron menores que la muestra control, esto debido a un proceso de división celular lento de Allium cepa en las células meristemáticas radiculares y menores en determinadas fases ${ }^{7}$. Estos valores del índice mitótico permiten evaluar la división celular y su relación con la presencia de agentes a los que se exponen los sistemas del ensayo interpretado como toxicidad mitótica (Tabla 1).

Cuando valores del índice mitótico son menores al control estarían manifestando en los organismos alteraciones por el agente al que se ha sometido ${ }^{8}$, lo cual indica la presencia de genotoxicidad, producida por sustancias tóxicas circulantes en estas aguas, también los índices de las fases profase y telofase fueron mayores, un caso particular en metafase donde el índice fue menor en la muestra control, en comparación con las muestras problemas, siendo la prueba ANOVA $(F(11)=4,30 ; P=0,0440)$, lo que indicó diferencias significativas en las muestras, y la prueba LSD evidenció diferencias entre la muestra control y las muestras de la Avenida Primera y Los Garzones, esto asociado a que las células que se dividieron presentaron anomalías, el análisis en los índices cuando se compara con el control podría manifestar una demora en los estadios de esta fase como exposición a sustancias que interfieren en la dinámica de polimerización y despolimerización del huso mitótico? ${ }^{7}$.

Al estar los IM por encima de los valores del control y ser estimulados por la división celular, estaría determinando que las células presenten daño, produciendo un exceso de división, contribuyendo a la formación de aberraciones ${ }^{9}$.

Los daños que se producen en el proceso de división celular de tipo nuclear o cromosómico pueden estar asociados a diversas sustancias físicas o químicas que alteran este proceso, las actividades antropogénicas tienen un impacto sobre la biota como la invasión informal sobre ecosistemas acuáticos, causando contaminación de fuentes hídricas ${ }^{10}$, Identificándose para este estudio alteraciones en los núcleos y fases mitóticas de las células de Allium cepa en cada muestra problema provenientes del río, que indicaron diferencias significativas con $(P=0,0130)$, afectado por factores como plaguicidas utilizados en la agricultura, aguas residuales derivadas de usos domésticos e industriales, etc. Reportes por ${ }^{11}$, indicaron que las exposiciones a ciertos plaguicidas causan daños al ADN, considerados como contaminantes emergentes en aguas residuales.

Los daños causados por estos compuestos se reflejan en el estudio realizado por ${ }^{12}$, sobre Índices de calidad ambiental de aguas del Arroyo Caañabe mediante la metodología de 
179

Allium test, donde los puntos de muestreo indicaron p-valor $<0,05$, mostrando efectos citotóxicos del agua en células meristemáticas de Allium cepa que no resultaron ser significativas.

Las alteraciones observadas fueron escasas para el punto Jaraquiel, lugar retirado del casco urbano de Montería donde el vertimiento de sustancias dañinas es poca sin embargo los factores como extensas lluvias provocan inundaciones, que atraen desechos contaminantes que pueden estar afectando a distintos organismos que estén expuestos de forma directa o indirecta a estas aguas, ocasionado la presencia de genotoxicidad por alteraciones en su material genético ${ }^{13}$. En este caso se identificaron alteraciones en Allium cepa como metafase con alteración numérica, anafase irregular con cromosomas desprendidos, célula con núcleo doble.

El crecimiento urbano que se presenta a orillas del río Sinú afecta el cauce por descargas residuales provenientes de usos doméstico u otras actividades como lavado de vehículos, al igual que la utilización de plaguicidas, pesticidas para la mejora de cultivos, y la construcción de obras aledañas, como casas, fabricas etc., siendo un factor de contaminación en estas aguas, lo que produjo irregularidades en la división celular de Allium cepa en las fases mitóticas de las muestras de aguas del punto Avenida calle 37. En resultados similares fueron reportados ${ }^{14}$, donde se evaluaron alteraciones de daños mitóticos en raíces de Allium cepa con muestras de agua superficial del río Pitimbu en Brasil.

En Los Garzones fueron evidentes un gran número de anomalías, indicando que este punto mostró un alto grado de genotoxicidad, debido a la acumulación de sustancias tóxicas presentes en esta zona que han venido siendo arrastradas por el caudal, las diferentes anomalías encontradas como presencia de micronúcleos (Figura 2), resultados similares a estudios reportados sobre ecosistemas acuáticos por ${ }^{15}$, donde las muestras presentaron micronúcleos en las aguas del río Guandú en Brasil, lo que indica la presencia de contaminantes con potencial citotóxico y genotóxico. Los micronúcleos aparecen cuando se fragmentan cromosomas durante la división de las células y son incapaces de unirse al huso mitótico, adquiriendo la forma de núcleos pequeños, por consecuencia de sustancias con efectos mutagénicos ${ }^{16}$.

Otros estudios como ${ }^{17}$ muestran los daños causados en el ADN por compuestos como el hipoclorito de sodio en Allium cepa identificándose aberraciones como anafase con puente, rompimiento de cromosomas en metafase, malformación de células, núcleos 
180

periféricos y telofase con pérdida de cromosoma, lo que promueve a que estos elementos puedan estar presentes en afluentes de ríos.

\section{Conclusiones}

El río Sinú de la ciudad de Montería se ve influenciado por sustancias contaminantes que generan cambios en el material genético indicando la presencia de genotoxicidad, como lo demostró el estudio con Allium cepa presentando alteraciones en su proceso de división celular en las fases mitóticas, por lo tanto, este estudio permite alertar sobre el problema evidente para los organismos que se encuentra habitando en este ecosistema ya que se podrían ver afectados.

\section{Agradecimientos}

Al Laboratorio de Genética y al Laboratorio de Microscopía del Centro de Investigación Piscícola CINPIC de la Universidad de Córdoba.

\section{Referencias bibliográficas}

1. Barbério, A. Bioassays with plants in the monitoring of water quality. In: Chowdhury WERK, editor. Water treatment: InTech; 2013. p. 317-34.

2. Acosta, K. La economía de las aguas del río Sinú, Documentos De Trabajos Sobre Economía Regional, 2013. 194, 121-122.

3. Aguilar, E. El Parque Lineal la Ronda del Sinú: dándole la cara al río. 2013. http://www.institut-gouvernance.org/fr/experienca/fiche-experienca-33.html, Consulta: enero de 2018

4. Tedesco, SB., Laughinghouse, IV HD. Bioindicator of genotoxicity: The Allium cepa test. In: Srivastava JK, editor. Environmental Contamination: InTech 2012. p. 137-56

5. Firbas P. Use of Allium test for purpose of establishing quality of water. 2011. https://www.flaska.si/files/original/756.pdf. Consulta; enero de 2018

6. Fiskesjö, G. The Allium test as a standard in environmental monitoring. Hereditas.1985.102:99-112. http://dx.doi.org/10.1111/j.1601-5223. 1985.tb00471.x

7. Andrioli, N. Evaluacion de agentes quimicos con potencial genotóxico en células meristematicas de Allium cepa.2011. Tesis Doctoral. Universidad de Buenos Aires, Argentina

8. Liman, R., Ciǧerci, I.H., Akyıl, D. et al. Determination of genotoxicity of fenaminosulf by Allium and comet tests. Pesticide Biochemistry and Physiology 2011;9961-64. https://doi.org/10.1016/j.pestbp.2010.10.006

9. Hoshina, M.M. Evaluation of a possible contamination of the waters of the Claro RiverMunicipality of Rio Claro, part of the Corumbataí River Basin, with the mutagenicity tests 
181

10. using Allium cepa 2002. Tesis Licenciatura en Ciencias Biológicas. State University of São Paulo, Rio Claro, SP.

11. Pinedo, J. Urbanización Marginal e Impacto Ambiental en la Ciudad de Montería. 2012. Tesis Doctoral en urbanismo. Universidad Politécnica de Valencia. España.

12. Gil, M., Soto, A., Usma, J. et al. Emerging contaminants in waters: effects and possible treatments. Producción más Limpia 2012;7(2):52-73.

13. Arias, TL., Peralta VF., de Diana DMF. et al. Índices de calidad ambiental de aguas del Arroyo Caañabe mediante tests microbiológicos y ecotoxicológico. Revista Ambiente \& Água. 2016;11(3):549-65. http://dx.doi:10.4136/ambiagua.1703

14. Leme, DM., Marin-Morales, MA. Allium cepa test in environmental monitoring: a review on its application. Mutation Research 2009;682(1):71-81. http://dx.doi: 10.1016/j.mrrev.2009.06.002

15. Monte, L., Graças, M., Regina, S. et al. Cytotoxic and genotoxic potential of Surface wáter from the Pitimbu river, northeastern/ RN Brazil. Genetics and Molecular Biology 2007;30(2): 435-441. http://dx.doi.org/10.1590/S1415-47572007000300023

16. Gomes, J., Tamara, J., Moreira, V. et al. Induction of cytotoxic and genotoxic effects of guandu river waters in the Allium cepa system. Revista Ambiente \& Agua; 2014;10(1):4858. http://dx.doi.org/10.4136/ambi-agua. 1487

17. Fenech, M. The in vitro micronucleus technique. Mutation Research, 2000; 455(1):81-95. https://doi.org/10.1016/S0027-5107(00)00065-8

18. Restrepo, R., Reyes, D., Ortiz, M.C. et al. Aberraciones cromosomales en bulbos de cebolla Allium cepa inducidas por moléculas híbridas 4-aminoquinolínicas. Universitas Scientiarum; 2012.17(3), 253-261. https://doi.org/10.11144/javeriana.SC17-3.aceb

19. Pardo $P, E$. , Cavadía M,T., Alvarino,G.2015.Análisis de la diversidad genética de la paloma domestica (Columba livia) en Bogotá, Colombia utilizando genes que codifican la coloración y diseño del plumaje. Bistua:Revista de la Facultad de Ciencias Básicas.13(1):35-45 
Tabla1. Valores de los Índices mitóticos e índices de las fases mitóticas en las células meristemáticas radiculares de Allium cepa.

\begin{tabular}{lccccc}
\hline \multirow{2}{*}{ Índices } & \multicolumn{5}{c}{ Muestras } \\
& Control & Jaraquiel & $\begin{array}{c}\text { Avenida } \\
\text { primera }\end{array}$ & Garzones & DS \\
Mitótico (\%) & 9.36 & 6.66 & 9.1 & 7.73 & 2.25 \\
Profase (\%) & 44.12 & 36.92 & 32.79 & 33,79 & 14.16 \\
Metafase (\%) & 16.95 & 21.9 & $27.66^{*}$ & $26.28^{*}$ & 5.56 \\
Anafase (\%) & 19.82 & 17.31 & 21.3 & 24.03 & 5.86 \\
Telofase (\%) & 21.08 & 19.46 & 19.52 & 15.86 & 10.10 \\
\hline
\end{tabular}

${ }^{\star}$ Indica diferencia significativa (P- valor $\left.\leq 5 \%\right)$

Tabla 2. Alteraciones celulares en las células meristemáticas radiculares de Allium cepa.

\begin{tabular}{|c|c|c|c|c|c|c|c|c|c|c|}
\hline \multirow{2}{*}{ Muestras } & \multicolumn{9}{|c|}{ Alteraciones } & \multirow{2}{*}{ DS } \\
\hline & MAN & ARC & $A P$ & CDN & MN & MRC & AAE & TP & $\mathrm{Al}$ & \\
\hline Control & - & - & - & - & - & - & - & - & - & - \\
\hline Jaraquiel & 6 & 3 & - & 1 & - & - & - & - & - & 2,82 \\
\hline La 37 & 13 & 7 & - & 6 & - & - & - & - & 1 & 6,53 \\
\hline Garzones & 15 & 18 & 1 & 10 & 14 & 1 & 1 & 1 & 1 & 10,07 \\
\hline
\end{tabular}




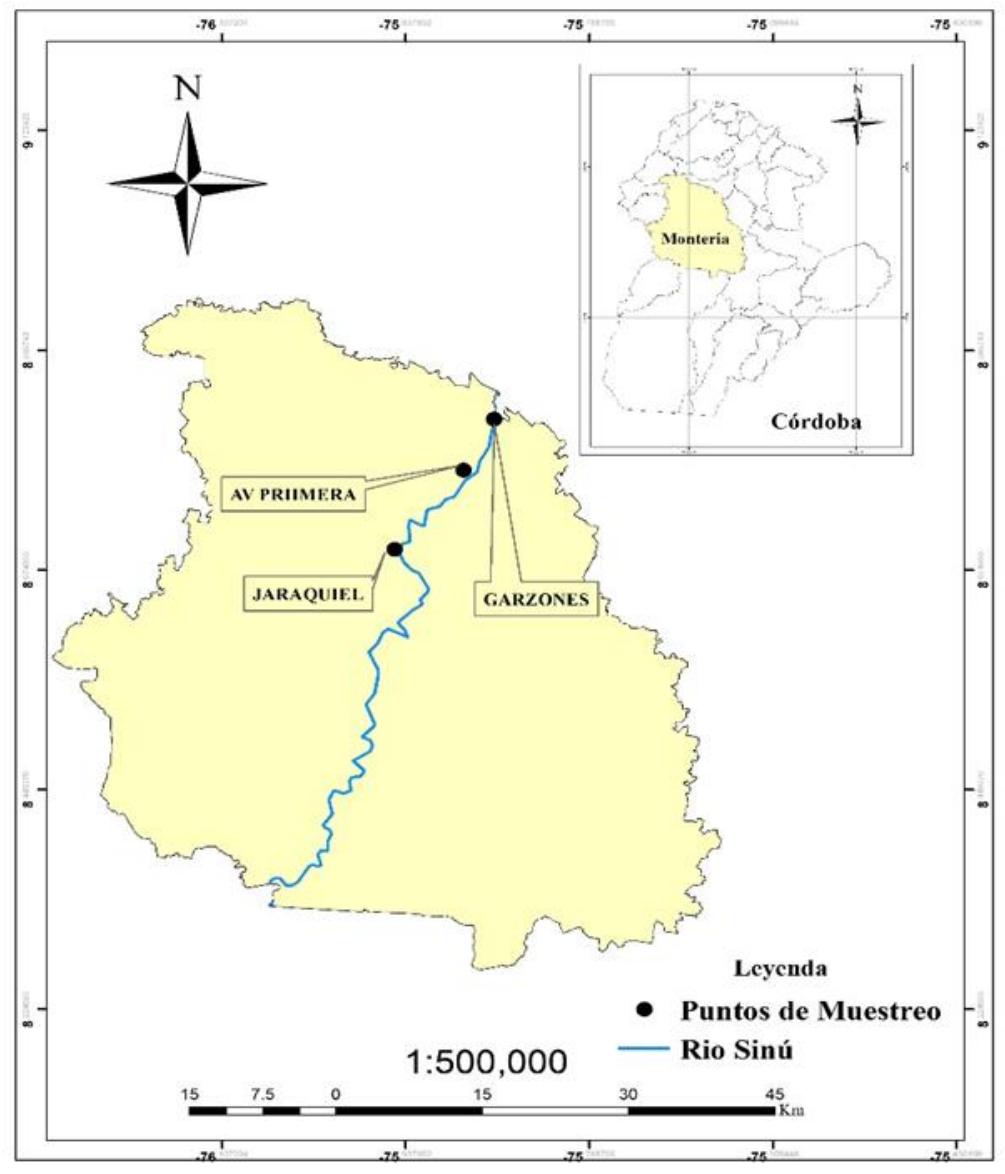

Figura 1. Mapa de ubicación de las zonas de muestreo del rio Sinú en la ciudad de Montería - Córdoba 

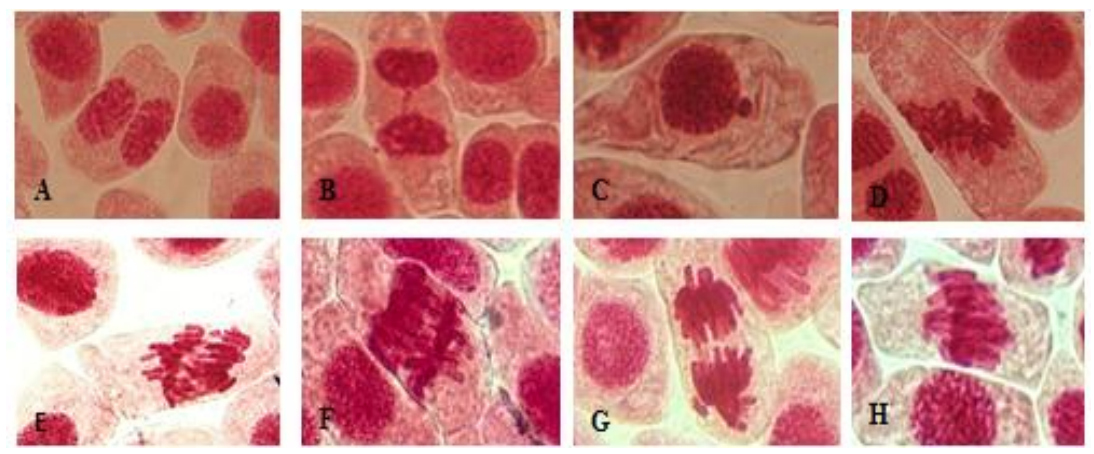

Figura 2. Alteraciones celulares observadas. A-célula con núcleo doble, B-Telofase con puente, C- Micronúcleos, D-Metafase con cromosomas desorientados, E-, anafase con alteración en el eje, F- Anafase irregular, G- anafase con rotura cromosómica- metafase con alteración numérica

*Para citar este artículo: Cavadía M T.I.; Roche M.M; Rosalyn Romero A.R. Estimation of the genotoxicity of the Sinú river through to bioassay with Allium cepa I. in Montería, CórdobaColombia. . Revista Bistua.2018.16(1):174-184.

+ Autor para el envió de correspondencia y la solicitud de las separatas: Cavadía M .T.I. Universidad de Córdoba, Facultad de Ciencias Básicas, Programa de Biología, Montería- Colombia. epardop@correo.unicordoba.edu.co

Recibido: Enero 30 de 2017

Aceptado:Febrero 12 de 2018 